\title{
Effectiveness of Peer-Led Interventions for Adolescents: A Meta Analysis of Smoking Prevention Program
}

\begin{abstract}
Babita Thapa ${ }^{1}$
Abstract

Background and Aims: Peer-led intervention is one of the beneficial approaches for preventing adolescents from health risk behavior. So, peer-led intervention may help to prevent adolescents from smoking cigarettes but impact of such intervention has not been studied well. Therefore, this study was conducted to investigate the effect of peer-led intervention to prevent adolescents from smoking behavior.

Methods: Pub Med, PschyINFO, EMBASE, Google Scholar and Cochrane Library were searched from March to June, 2016, with set criteria in review protocol and only randomized control trials were included. Screening and data extraction were conducted and data from eligible studies were pooled.

Results: The number of studies included after full text review were three. Three eligible studies' data were extracted and further analyzed for risk of bias in Revman. ${ }^{5}$ Three studies targeting smoking behavior were pooled which represent 25,864 adolescents. Meta-analysis revealed that odd ratio of smoking was lower in those receiving peer-led intervention compared to those not receiving peerled intervention.
\end{abstract}

Conclusions: Peer-led intervention may be effective in prevention of cigarette smoking among adolescents but the studies included in the study are less in number.

Keywords: Adolescent, Smoking, Tobacco, Intervention, Prevent

\section{Introduction}

Smoking is one of the major health risk behaviors in adolescents. According to the World Health Organization, ${ }^{19}$ the prevalence of tobacco use among adolescents aged 13-15 years is $8.3 \%$ (female) and $18.2 \%$ (male) globally, 7.2 (female) and 21 (male) in South-East Asia, 13.8 (female) and 17 (male) in the United States. At the same time, 80 percent of more than 1 billion smokers worldwide live in low- and middle-income countries where the burden of tobacco-related illness and death is heaviest. ${ }^{19}$ Smoking is one of the leading causes of morbidity and mortality. ${ }^{18}$ In adolescents, smoking causes asthma and other respiratory diseases, and is well known as a risk factor for lung cancer in later life. ${ }^{15}$ Both the government commitment and public awareness interventions have shown promise in preventing smoking in adolescents worldwide.

\footnotetext{
${ }^{1} \mathrm{PhD}$ Scholar, School of Education, Kathmandu University, Nepal.

E-mail Id: babithapa@yahoo.com

Orcid Id: http://orcid.org/0000-0003-3457-6238

How to cite this article: Thapa B. Effectiveness of Peer-Led Interventions for Adolescents: A Meta Analysis of Smoking Prevention Program. Ind J Youth Adol Health 2017; 4(1): 16-25.
}

Digital Object Identifier (DOI): https://doi.org/10.24321/2349.2880.201704

ISSN: $2349-2880$ 
Over 1.3 billion people, or $18 \%$ of the world's population, are protected by comprehensive national smoke-free laws. ${ }^{19}$ Despite efforts in smoking prevention, smoking behavior in adolescents has been increasing and it is considered as one of the major threats for adolescent health. According to the WHO, in Nepal, the prevalence of current tobacco use among adolescents aged 13-15 years is 5.3 (female) and 13 (male) in 2007 which increased to 16.4 (female) and 24.6 (male) in 2011.

Ninety percent of smokers have started smoking by the age of 18 and 99 percent have started by the age of $26{ }^{18}$ This indicates the importance of smoking prevention programs or interventions during adolescence. Peers are major social agents who influence the adolescents' behavior. They have similar values, ages, status and behaviors, and can share information and teach each other. So, peer-led interventions are considered as one of the major intervention strategies to reduce the health risk behavior among adolescents worldwide.

Peers have great influence on positive and negative behavior of the adolescents. In peer-led interventions, peer education is used to influence peers in a positive way to improve health risk behavior. ${ }^{6}$ Peer educators are generally of similar or slightly older age than the students receiving educational program. ${ }^{4}$ Peer educators are more likely to influence peers because they are usually less judgmental and credible to their peers and such intervention involving peers can access the hidden populations. ${ }^{20}$ In most of the interventions, peer educators may act as a role model, innovator, and educator to change the health risk behavior of adolescents and solve health problems in partnership.

The peer-led approach is quite popular in major health interventions related to HIV and AIDS, family planning and drug prevention. Peer education program in HIV prevention in low- and middle-income countries demonstrated some success in changing community attitudes and norms but effects on other sexual behaviors and STI rates were equivocal. ${ }^{16}$ Both peer-led and adult-led interventions generally have their own place in effective sex education but the main challenge is which area should be dealt with peer-led intervention to get maximum benefit. Peer-led education is less effective in communicating factual information on one hand but on the other hand it is more effective in dealing with teenage relationship and setting conservative norms. ${ }^{4}$ Peer-led interventions need more time to train peer and need more effort to fix the venue for peer education. ${ }^{3}$
There are a number of types of peer-led interventions to prevent adolescents from smoking behavior. Each of these peer-led interventions uses different theory and principles. So, in this context, it is important to provide best possible evidences on peer-led interventions out of many interventions applied in smoking prevention programs worldwide. In this meta-analysis, peer-led intervention means all the smoking prevention interventions which involved peers to delivery of smoking prevention activities directly or indirectly to adolescents.

Till date, most of the systematic reviews were done on teacher-led school-based programs and only very few systematic reviews were conducted on peer-led interventions. Most of the peer-led reviews were conducted to find the effect of peer-led interventions on multiple risk behaviors including alcohol and drug besides smoking behavior. The main objective of this study is to assess the effectiveness of peer-Led interventions for preventing adolescents (aged 13-19) from smoking. So, the set hypothesis for this study was "Peer-led interventions are more effective in preventing adolescents from smoking."

\section{Methods}

The protocol of systematic review is prepared on the basis of questions provided by the university. Then, it was sent to the university professor for approval. After getting approval on protocol from the university, the review started. The primary objective of the review was to identify and review the effect of peer-led intervention in preventing adolescents aged $10-19$ years from smoking behavior. This age group was chosen based on the WHO definition of adolescents and because many young people start smoking behavior below the age of 20 .

\section{Criteria for Considering Studies}

The review included only randomized control trials (RCTs). Only articles published after 2005 were included. Moreover, papers published in non-English language and grey literature were excluded. Only published journal papers were included in the study. The studies were considered on the basis of population, intervention, comparison, outcomes and context (PICOC).

Population (P): All the studies that included young people aged between 10 and 19, who were currently studying in school/college were included but out-ofschool or college adolescents were not included in this meta-analysis. 
Intervention (I): Peer-led intervention means all the smoking prevention interventions, which involved peers in the delivery of smoking prevention activities directly or indirectly to adolescents.

Comparison( $\mathrm{C})$ : The studies assessed the effectiveness of the interventions on the basis of comparison between intervention and control groups. The comparison was done on the basis of program intervention such as peer-led discussion and interaction program after peer-led intervention such as peer-led video show, lecture, poster, pamphlets, advocacy, etc.
Interventions with less than 6 months' follow-up period were excluded in the selection process.

Outcomes (0): The primary outcome was smoking prevalence, which was measured as the number of new smokers and/or number of quitters. Only studies that had at least 6 months' follow-up period were considered.

Context(C): Study included only peer-led interventions and it excluded multi-component interventions where one of the components was peer-led intervention.

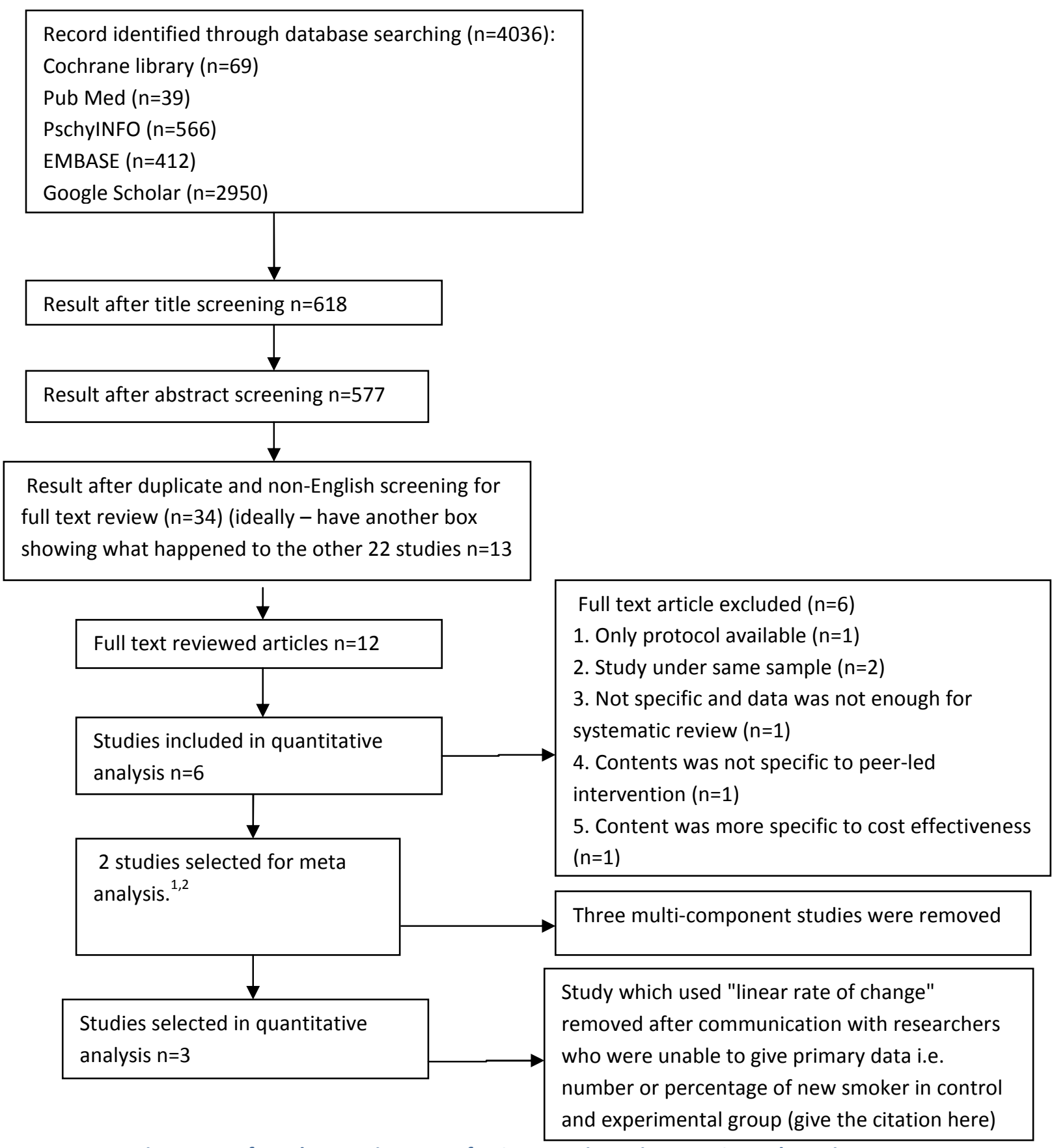

Figure 1.Preferred Reporting Items for Systematic Reviews PRISMA Flow Diagram 


\section{Search Methods for Identification of Studies}

A systematic literature search was performed to identify published randomized control trials (RCTs). Keywords and synonyms were identified through Google Scholar and Pub Med limited search. The keywords and synonyms identified were used in extensive literature search in respective database. The basic search was done with major key words "adolescent," "peer,*" "smoking," "tobacco," "prevent, *" "intervention."

The search was done systematically by using four electronic databases, namely, "Pub Med," "PsycINFO," "EMBASE" and "Cochrane library." Furthermore, search was also carried out through search engine "Google scholar." Boolean operators were used during searching process. Search process was presented in PRISMA's recommended flow chart. ${ }^{13}$ Duplicates were removed from total identified articles from above mentioned database and search engine step by step.

Identified titles were reviewed and relevant articles were selected for further screening or abstract review. Screening of abstract was done based on predefined inclusion and exclusion criteria. After reviewing abstract, full articles from relevant studies were retrieved. Critical appraisal of full text articles was carried out among the selected articles in abstract review. Reasons for exclusion of full text articles were presented in flow chart in each step. Articles which met the inclusion criteria were retained for full analysis. The reference lists of selected articles were further searched for more relevant articles. Any confusion while selecting articles was resolved by discussion with study mentor of this meta-analysis.

\section{Critical Appraisal}

Risk of bias was assessed using the Cochrane tool. ${ }^{9}$ Cochrane tool was used to check the low, high and unclear risk of bias. The major risk of bias considered in the reviews were selection bias (random sequence generation and allocation concealment), performance bias (blinding of participants and personnel), detection bias (blinding of outcome assessment), attrition bias (incomplete outcome data), reporting bias (selective reporting) and other biases. Risk of bias analysis was done for three studies, namely, study $1,{ }^{2}$ study $2,{ }^{1}$ and study $3,{ }^{5}$ as shown in Fig. 2.

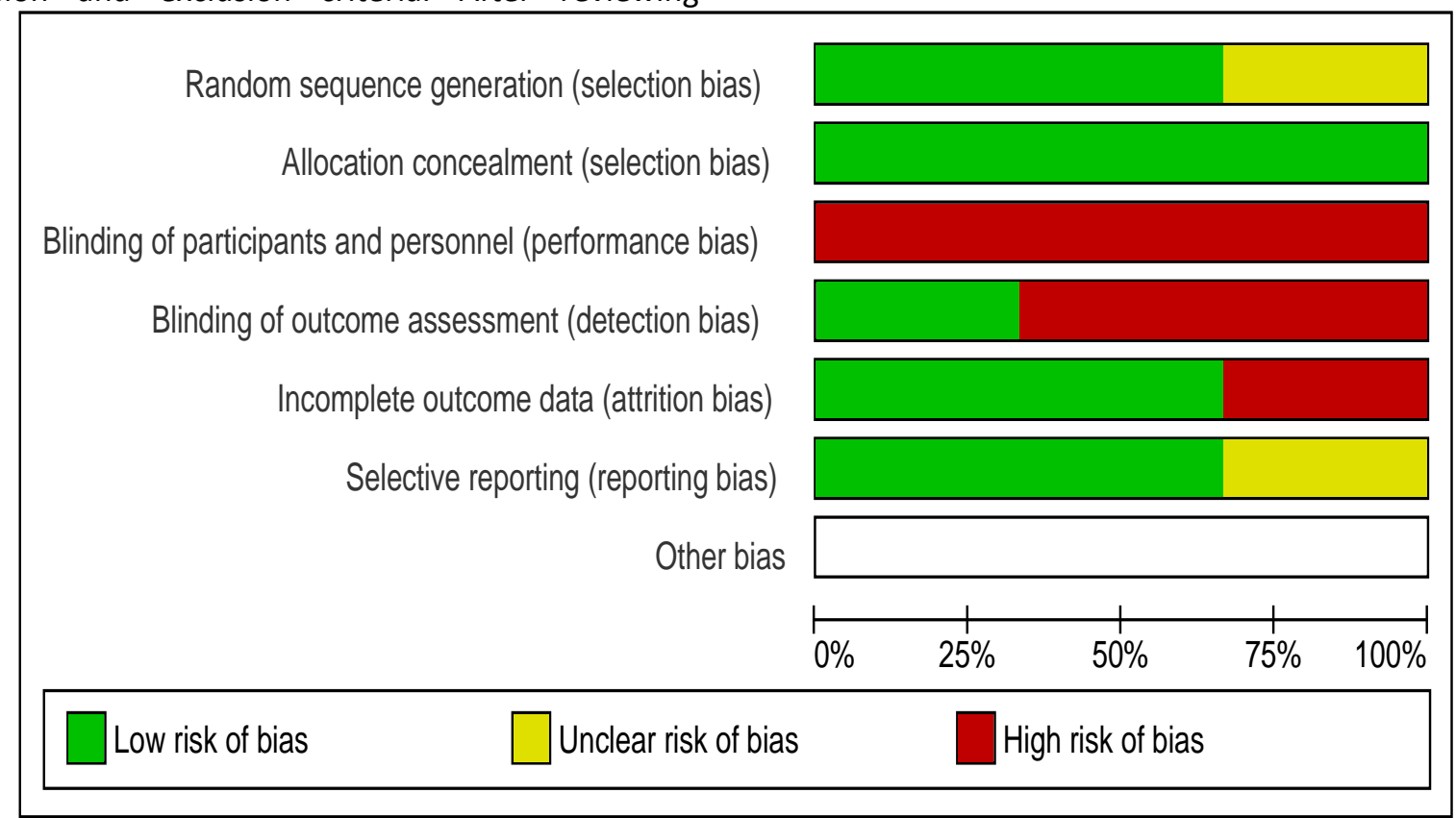

Figure 2.Review Author Judgments about Each Risk of Bias Item Presented as Percentages across All Included Studies

\section{Selection Bias}

\section{Random Sequence Generation}

Altogether 10 schools were randomly assigned and by tossing coins, the two groups were randomly assigned as experimental and control groups so there is low risk of selection bias due to random sequence generation in study 1 . Study 2 also showed low risk of selection bias as in this study stratified block randomization was done.

Study 3 showed unclear random sequence generation process but it highlighted about group randomized trial. "As a means of ensuring representativeness, schools within each city were matched according to type of school and then randomly assigned to receive the 
tobacco use intervention program." The article did not mention the clear procedure of randomization.

\section{Allocation Concealment}

In study 1, participants and Investigators could not foresee the assignment because all the names were kept in a box and the investigator took the number by lottery and two created groups were named control and experiment group by tossing coin so there is low risk of selection bias due to allocation concealment. Actual blinding was not possible in this study. The study 2 stated that the schools were allocated using randomly ordered list of schools for each stratum. It had low risk of selection bias due allocation concealment. For conceal allocation, another investigator was at a different location and was unaware that the school was next to be randomized and random number generator was used to establish the group allocation of next school, which was communicated to first investigator by telephone. in study 3, which stated that "Student response by the use of unique identification tag that was not recognizable to students or school staff over time so allocation concealment was probably done and therefore had low risk of selection bias."

\section{Performance Bias}

If the participants were asked their view whether they will stop smoking in future, many of them might say yes during self-administered data collection; this might not be due to intervention effect but due to the participants' knowledge about the intervention intention so there was high risk of performance bias due to lack of blinding of participants and personnel in study 1. As in previous study, study 2 also showed actual blinding of participants and personnel was not possible in this study so it has high risk of performance bias. If the participants were asked their view regarding whether they will stop smoking in future as the intervention itself named as "A stop smoking in school trial." Many of them might say yes during selfadministered data collection; this might not be due to intervention effect but due to the participants' prior knowledge about the intervention intention.

Study 3 showed high risk of performance bias. Students, peers and teachers were aware about program intention so, in self-administered questions when asked about their intention and prevalence they will answer as they wanted to stop smoking so, blinding of participants and personnel in this case was not possible. No biochemical analysis of tobacco use was done due to expense of obtaining and analyzing such data.

\section{Detection Bias}

Study 1 showed high risk of detection bias as blinding of outcomes' assessment was not possible. Double blinding was not possible as the peer educators and trainer were trained and got manual. Students expressed their view in self-administered questionnaire. So, participants might have had knowledge about intervention intention; students subconsciously answered questions according to program intention. They had prior knowledge of getting educational intervention.

Study 2 had low risk of detection bias due to blinding of outcome assessment. "Comparison of self-reported data and concentration of salivary cotinine shows $1 \%$ of students who reported not smoking had salivary cotinine concentration greater than $15 \mathrm{ng} / \mathrm{mL}$ at 1 year follow up." This indicates the study not only collected outcome assessment through self-administered questionnaire where probability of answering on the basis of intervention intention was reduced by cross validation with saliva test.

Study 3 did not mention about double blinding. Double blinding was not possible as the peer educators and supervisor teachers were trained and got standardized protocol on how to educate students. Students expressed their views in self-administered questionnaire during baseline, midline and end line. So, participants had knowledge about intervention intention, so students generally subconsciously answered according to program intention. Blinding of outcome assessment might not be possible. So, this study had high risk of detection bias.

\section{Attrition Bias}

Study 1 had low risk of attrition bias due to complete outcome data. "Dropout rates were similar $(p>0.05)$ in both conditions, $11 \%$ in experiment group and $9.8 \%$ in control group, which was mostly by absenteeism and change of schools by some students. Similarly, study 2 also showed low risk of attrition bias." Two schools withdrew after randomization, one from the control group and one from the intervention group because of changes in decision by school management and replacement was done by each school one from each strata in the list. In one-year follow-up, $93 \%$ students in control group and $96 \%$ students in intervention group participated. In two-year follow-up, $90 \%$ students in control group and $94 \%$ students in intervention group participated which showed the dropout percentage seems not so high. Study 3 showed high risk of attrition bias. Follow-up data from two of Delhi schools (one 
intervention and one control school) was not obtained due to time constraint at these schools. Three additional schools in Delhi (two control and one intervention school) would not allow their 10th graders to be surveyed because of ensuing exams

\section{Reporting Bias}

Study 1 had unclear risk of reporting bias. It measured behavioral outcomes and the secondary outcomes like attitudes, social influence, self-efficacy and intention to smoke in future but study 2 showed low risk of reporting bias due to selective reporting. It reported that outcomes as expected in four major time periods in baseline, immediately after intervention, at one year follow up and at two year follow up and measured outcome data were reported as planned. Study 3 reported outcome data of three major periods, i.e., before the beginning of intervention, midpoint of intervention and after the completion of intervention, so it had low risk of reporting bias.

\section{Data Extraction and Analysis}

Data were extracted by using Review Manager 5.3 (RevMan) for each included study. Data were extracted for all included studies on study design, age of participants, gender of participants, intervention duration and follow up, brief overview of peer-led intervention and details of control group as in Table 1. Data were extracted for never-smoker. Both base line and follow-up data were collected for both intervention and control groups. Confusions were solved by discussion with study mentor.

\section{Results}

Figure 1 shows the total number of studies identified, screened and reviewed. It shows the reason for exclusion of each excluded study after review of full articles. A total of 4036 articles were identified by database search and through Google Scholar. The total number of articles after title screening were 618 which were further screened by abstract review. Altogether 577 articles were identified for abstract review. After removal of duplicate and non-English articles in abstract review process, a total of 34 articles were selected. From 34 articles, finally 12 articles were selected for full text review. Six articles were selected after full text review of 12 articles. At this stage, articles were excluded mainly due to the following reasons: only protocol available, study were conducted in same sample, not enough data was available for systematic review, content was not specific to peer-led interventions and content was more specific to cost effectiveness. After further full review of six articles, three multi-component studies were excluded. Finally, three studies were selected for quantitative analysis. Out of three articles, two studies used the number of smokers as measurement units but one study used linear rate of change to calculate intervention effect. Authors of these articles were contacted for primary data to calculate odd ratio. Authors were unable to provide primary data of this study. Finally, two studies were selected for meta-analysis.

Table 1 summarizes the characteristics of the included studies. Selected three studies were conducted in Romania, UK \& Wales, and India. Out of three studies the first study included adolescents aged between 13 and 14 years, while the second study included students aged between 12 and 13 and the third study included students from grade 6 to 8 without mentioning their age. All included studies were targeted to tobacco smoking. Included studies were school-based intervention, which did not cover out-of-school adolescents. All the studies were heterogeneous in nature where duration of intervention varied from one year to three years. Follow-up period for all three studies was nine months to three years. Meta-analysis of the studies' outcomes from nine months to twelve months was conducted.

The post test results indicated weekly smoking on set was 4.5 in experimental group versus 9.5 percent in control group - more than double the number of new smokers in control group than in experimental group. The first included study showed that more than double the number of new smokers in control group than in experimental group.

In the second study, the odd ratio of being a smoker in intervention compared with control school was 0.75 (95\% Cl, 0.55-1.01) immediately after intervention ( $n=9349$ students), 0.77 (0.59-0.99) at one-year followup ( $n=9147$ students) and 0.85 (0.72-1.01) at two-year follow-up ( $n=8756$ students). Therefore, the study showed that intervention was fruitful in reducing smoking prevalence in adolescents in experiment group.

In the third study, cigarette smoking linear rate of change was 1.37 (95\% Cl, 0.72-2.02) in control group and $0.46(95 \% \mathrm{Cl},-0.19-1.11)$ in intervention group. The study also showed that more students in control group smoked cigarette and bidis than students in intervention group. 
Table 1.Table of Included Studies

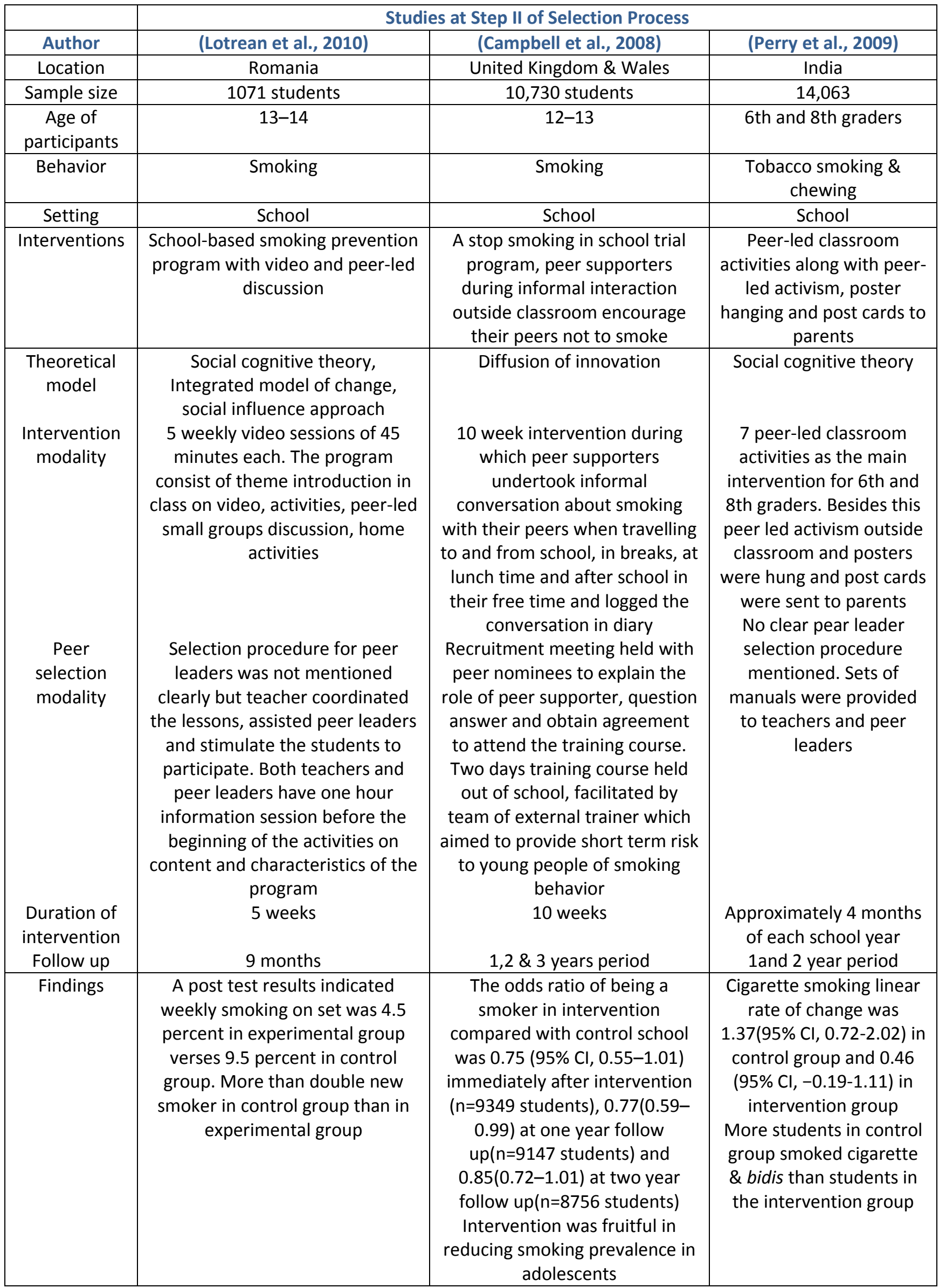




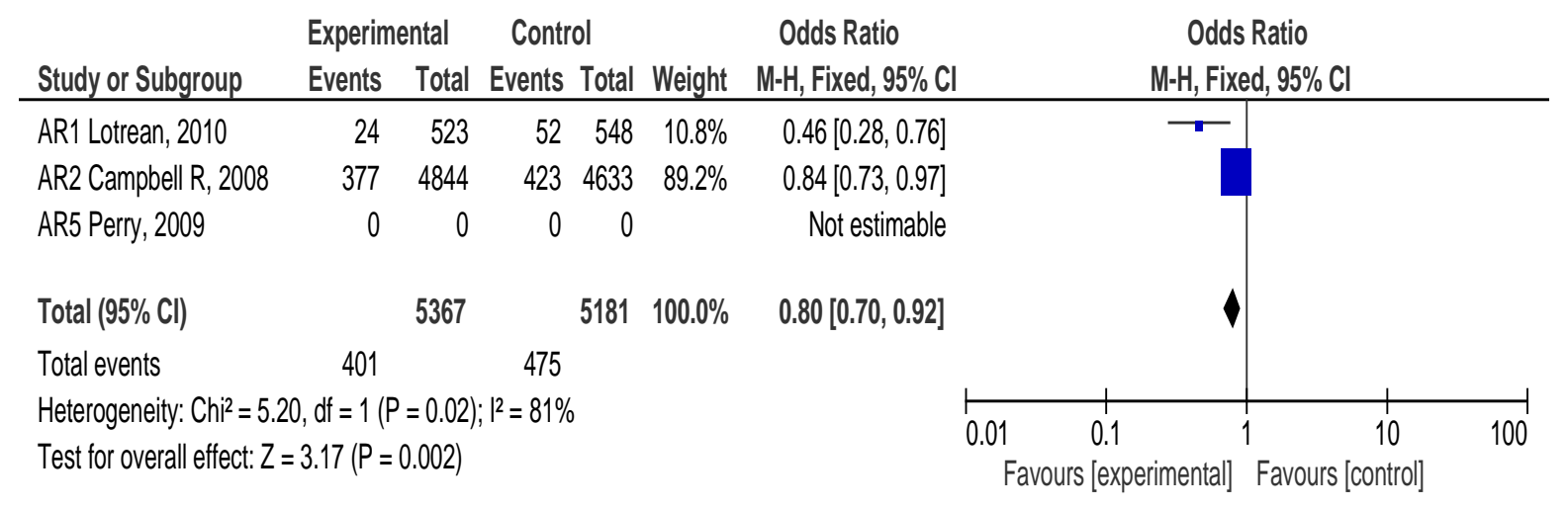

Figure 3.Forest Plot Showing Results for Effectiveness of Smoking Prevention Program in Experimental and Control Groups

Figure 3 shows the effectiveness of peer-led interventions by showing number of new smokers in follow-up period in experimental and control group. Data were pooled for two main studies. In the first study AR1, number of new smokers is higher in control group than in experimental group with odd ratio $0.46(0.28$, $0.76)$ at $95 \% \mathrm{Cl}$ in fixed-effect model. Similarly, in second study AR2, number of new smokers is lower in experimental group with odds ratio $0.84(0.73,0.97)$ at $95 \% \mathrm{Cl}$ in fixed-effect model. Though the sample size of the AR1 was small, the study demonstrates a significant effect favoring the intervention, which is greater than for AR2. The diamond of the forest plot shows that overall, peer-led intervention is an effective approach in smoking prevention for adolescents. The total effect shows odd ratio of $0.80(0.70-0.92)$ at $95 \% \mathrm{Cl}$. As the overall odds ratio is less than 1 , this indicates that such an intervention is effective in preventing adolescents to initiate smoking. Among the two studies, $\left.\right|^{2}=81 \%$, chi $2=5.20 \mathrm{df}=1(\mathrm{p}=0.02)$, which shows substantial statistical heterogeneity.

\section{Discussion}

In this study, the impact of peer-led intervention on smoking behavior is quantified. The findings of the study showed that peer-led intervention can play a significant role in prevention of smoking behavior among adolescents. The pooling of two main studies including 11,801 adolescents aged $12-14$ years suggests that number of new smokers in experimental or intervention group was less in comparison to number of smokers in control or the without-intervention group. The odds of becoming a new smoker were lower among those who received a peer-led intervention compared to control group $(\mathrm{OR}=0.80,95 \% \mathrm{Cl}=0.70-0.92)$. This result is based on only two studies, as the third study that was relevant reported data using a different method. Importantly, the results of the third study are in broad agreement with the meta-analysis, further strengthening the findings of this systematic review.

The systematic review of the studies suggested that peer-led interventions may be effective in preventing smoking behavior among adolescents but the evidence base is limited because of the high heterogeneity in included studies. Similarly, one of the systematic reviews on effectiveness of peer-led interventions to prevent tobacco, alcohol and/or drug use among young people aged 11-21 years also highlighted that most of the included studies on peer-led intervention were of low quality small studies, which limited the evidence base of review. ${ }^{3}$

\section{Limitation of the Study}

The major limitation of this study was heterogeneity of the data due to differences in effect size, intervention modality and follow-up period. In this review, the process of peer-led intervention implementation was very different; one of the studies conducted used formal school setting for the intervention while other used informal out-of-class interaction for peer-led interventions which increased the heterogeneity among included studies. The scope of analysis was also limited by exclusion of one of the major studies with sample size of 14,063 because the authors of the study were unable to provide data on the number of new smokers in intervention and control groups. The authors used linear rate of change and base line data for control and intervention group.

Furthermore, the study was limited due to exclusion of many studies due to their multi-components nature, where the peer-led intervention was one of the components besides other components. The study was conducted by single author as course assignment. The risk of bias might be higher in this study in comparison to other studies in which screening, critical appraisal 
and data extraction is done in duplicate. However, inclusion of studies was carried out by getting consensus from the mentor of this study.

\section{Acknowledgments}

This work was carried out as a partial fulfill of PhD course of Kathmandu University, Nepal. The author is grateful to Dr. Tim Schultz, Research Fellow and Postgraduate Coordinator, Adelaide Nursing School, University of Adelaide, Australia, for his guidance and support as a mentor of the study. The author is also thankful to Dr. Mana Prasad Wagle (Professor \& PhD supervisor, Kathmandu University) for his continuous support and valuable comments. The guidance and valuable comments from Dr. Maheshnath Parajuli (Dean, School of Education, Kathmandu University), Dr. Bal Chandra Luitel (Associate Dean, School of Education, Kathmandu University) and Dr. Prakash Chandra Bhattarai (Assistant Professor, School of Education, Kathmandu University) are also gratefully acknowledged.

\section{Conclusion}

Only a few systematic reviews have been conducted in the past on the effectiveness of peer-led interventions as part of a multi-component intervention in relation to risk behavior in adolescents, but none have examined the effectiveness of peer-led interventions on their own for smoking in adolescents. This study has found that peer-led interventions are effective in preventing smoking behavior among adolescents; however, the scope of this study is limited by its total small sample size and high heterogeneity among included studies. Therefore, to draw the conclusion on effectiveness of peer-led interventions on smoking behavior of adolescent, more robust and rigorous randomized control trials in wide range of geography and adolescent population was needed. Moreover, more homogenous studies and large number of such studies can add more value to draw the conclusion on effectiveness of the peer-led intervention in reducing risk behavior among adolescents. Therefore, future randomized control trials with homogenous program modality can help to inform future peer-led interventions strategy and program implementation in reducing risk behavior among adolescents.

\section{Conflict of Interest: Nil}

\section{References}

1. Campbell R, Starkey F, Holliday J et al. An informal school-based peer-led intervention for smoking prevention in adolescence (ASSIST): A cluster randomised trial. The Lancet 2008; 371(9624): 1595-1602.

2. Lotrean LM, Dijk F, Mesters I et al. Evaluation of a peer-led smoking prevention programme for Romanian adolescents. Health Education Research 2010; 25(5): 803-14.

3. Macarthur GJ, Sean H, Deborah MC et al. Peer-led interventions to prevent tobacco, alcohol and/or drug use among young people aged 11-21 years: A systematic review and meta-analysis. Addiction 2016; 111(3): 391-407.

4. Mellanby AR, Newcombe RG, Rees J et al. A comparative study of peer-led and adult-led school sex education. Health Education Research 2001; 16(4): 481-92.

5. Perry $\mathrm{CL}$, Stigler $\mathrm{MH}$, Arora $\mathrm{M}$ et al. Preventing tobacco use among young people in India: Project MYTRI. American Journal of Public Health 2009; 99(5): 899-906.

6. Raji MO, Abubakar I, Oche M et al. Using peer led health education intervention to improve in-school adolescents cigarette smoking related knowledge, attitude and behaviour in a North West Nigeria State. Health Science Journal, 2014; 8(4): 485-94.

7. Ye S, Yin L, Amico KR et al. Efficacy of peer-led interventions to reduce unprotected anal intercourse among men who have sex with men: $A$ meta-analysis. PLOS ONE 2014; 9(3).

8. Higgins J, Greens S. Cochrane Handbook for Systematic Reviews of Interventions version 5.1.0. Sussex, England: The Cochrane Collaboration 2011.

9. Keeney S, Hasson F, McKenna H. The Delphi Techniques in Nursing and Health Research. West Sussex: Blackwell Publishing 2011.

10. Raghav PR, Bhardwaj P. Experience of Delphi technique in the process of establishing consensus on core competencies. International Journal of Applied Basic Medical Research 2016; 6(3): 191-94.

11. Skaar NR. Development of Adolescent Exploratory and Risk Behavior Rating Scale (Unpublished dissertation). Minnesota: University of Minnesota 2009.

12. Stovold E, Beecher D, Foxiee R et al. Study flow diagrams in Cochrane systematic review updates: An adapted PRISMA flow diagram. BioMED Central 2014; open access.

13. Sustein R. Adolescent Risk-Taking and Social Meaning: A commentary. Chicago: University of Chicago 2008.

14. The Cancer Council. Tobacco in Australia: Facts and Issues. 2016. Retrieved October 30, 2016 from The health effects of active smoking: http:// www.tobaccoinaustralia.org.au/chapter-3-health- 
effects.

15. Tyndale EM, Barnett JP. Peer-led intervention to reduce HIV risk of youth: A review. Evaluation and Program Planning 2010; 33(2): 98-112.

16. University of IOWA. 2014 IOWA Youth Survey, State of IOWA Results. IWOA Department of Public Health. 2015.

17. US Department of Health and Human Services. The health consequences of smoking 50 years of progress: A report of the surgeon general. Atlanta, GA: US Department of Health and Human Services 2014.
18. WHO. World Health Organization, Media Center. June 2016. Retrieved July 15, 2016, from Fact sheets: http://www.who.int/mediacentre/fact sheets/fs339/en/

19. Ye S, Yin L, Amico KR et al. Efficacy of peer-led interventions to reduce unprotected anal intercourse among men who have sex with men: a metaanalysis. PLOS ONE 2014; 9(3): 1-11.

Date of Submission: $28^{\text {th }}$ Dec. 2016 Date of Acceptance: $04^{\text {th }}$ Mar. 2017 